\title{
Further results on the inverse along an element in semigroups and rings
}

\author{
Huihui Zhu ${ }^{\mathrm{a}}$, Jianlong Chen ${ }^{\mathrm{a}, *}$, Pedro Patrício ${ }^{\mathrm{b}}$ \\ ${ }^{a}$ Department of Mathematics, Southeast University, Nanjing 210096, China. \\ ${ }^{b}$ CMAT-Centro de Matemática and Departamento de Matemática e Aplicações, \\ Universidade do Minho, Braga 4710-057, Portugal.
}

\begin{abstract}
In this paper, we introduce a new notion in a semigroup $S$ as an extension of Mary's inverse. Let $a, d \in S$. An element $a$ is called left (resp. right) invertible along $d$ if there exists $b \in S$ such that $b a d=d($ resp. $d a b=b)$ and $b \leq_{\mathcal{L}} d$ (resp. $\left.b \leq_{\mathcal{R}} d\right)$. An existence criterion of this type inverse is derived. Moreover, several characterizations of left (right) regularity, left (right) $\pi$-regularity and left (right) $*$-regularity are given in a semigroup. Further, another existence criterion of this type inverse is given by means of a left (right) invertibility of certain elements in a ring. Finally we study the (left, right) inverse along a product in a ring, and, as an application, Mary's inverse along a matrix is expressed.
\end{abstract}

Keywords:

von Neumann regularity, Left (Right) regularity, Left (Right) $\pi$-regularity, Left (Right) *-regularity, Inverse along an element, Semigroups, Rings 2010 MSC: 15A09, 16E50, 16W99, 20M99

\section{Introduction}

Throughout this paper, $S$ is a semigroup. An element $a \in S$ is (von Neumann) regular if there exists $x$ in $S$ such that axa $=a$. Such $x$ is called an inner inverse of $a$. By $a\{1\}=\{x \in S: a x a=a\}$ we denote the set of all inner inverses of $a$. An arbitrary element in $a\{1\}$ is denoted by $a^{(1)}$.

\footnotetext{
${ }^{*}$ Corresponding author

Email addresses: ahzhh08@sina.com (Huihui Zhu), jlchen@seu.edu.cn (Jianlong Chen), pedro@math.uminho.pt (Pedro Patrício)
} 
The element $a$ is left (right) regular (see e.g. [2]) if there exists $x$ such that $a=x a^{2}\left(a=a^{2} x\right)$, and strongly regular if it is both left regular and right regular. It is left (right) $\pi$-regular (see e.g. [2]) if there exists $x$ such that $a^{n}=x a^{n+1}\left(a^{n}=a^{n+1} x\right)$ for a positive integer $n$. If $a$ is both left and right $\pi$-regular, then $a$ is strongly $\pi$-regular.

Let $*$ be an involution (anti-isomorphism of degree 2 ) on $S$, that is, the involution satisfies $\left(x^{*}\right)^{*}=x$ and $(x y)^{*}=y^{*} x^{*}$ for all $x, y \in S$. Let $a \in S$. We call $a$ left (right) $*$-regular if there is $x$ such that $a=a a^{*} a x\left(a=x a a^{*} a\right)$. A *-semigroup $S$ is called left (right) *-regular if all elements in $S$ are left (right) *-regular. If $x$ satisfies $a x a=a$ and $(a x)^{*}=a x$, then $x$ is a $\{1,3\}$ inverse of $a$. If $y$ satisfies $a y a=a$ and $(y a)^{*}=y a$, then $y$ is a $\{1,4\}$-inverse of $a$.

The standard notions of group, Drazin and Moore-Penrose inverse can be referred to the literature [4, 9]. Following [4], an element $a$ is Drazin invertible if and only if it is strongly $\pi$-regular. In particular, $a$ is group invertible if and only if it is strongly regular. It is well known that $a \in S$ is Moore-Penrose invertible if and only if $a \in a a^{*} S \cap S a^{*} a$ if and only if it is both $\{1,3\}$ and $\{1,4\}$-invertible. All these inverses, if they exist, are unique. We denote by $a^{\#}, a^{D}$ and $a^{\dagger}$ the group, Drazin and Moore-Penrose inverses of $a$, respectively.

Mary [6] recently defined a new generalized inverse in a semigroup $S$ called the inverse along an element. Motivated by [6], we introduce in section 2 below a new notion. An existence criterion of this type inverse is derived. Moreover, several characterizations of left (right) regularity, left (right) $\pi$ regularity and left (right) *-regularity are given in a semigroup. Also, we prove that $a \in S$ is Moore-Penrose invertible if and only if it is left $*$-regular if and only if it is right $*$-regular. In section 3 , another existence criterion of this type inverse is given by means of a left (right) invertibility of certain elements in a ring, and as an application, the formula of the inverse along a matrix is expressed.

\section{One-sided inverse along an element in semigroups}

Green's preorders in a semigroup [5] are defined as followed $\left(S^{1}\right.$ denotes the monoid generated by $S$ )

$a \leq_{\mathcal{L}} b \Leftrightarrow S^{1} a \subseteq S^{1} b \Leftrightarrow$ there exists $x \in S^{1}$ such that $a=x b$.

$a \leq_{\mathcal{R}} b \Leftrightarrow a S^{1} \subseteq b S^{1} \Leftrightarrow$ there exists $x \in S^{1}$ such that $a=b x$.

$a \leq_{\mathcal{H}} b \Leftrightarrow a \leq_{\mathcal{L}} b$ and $a \leq_{\mathcal{R}} b$. 
We next introduce a notion that is based on Green's preorders in a semigroup.

Definition 2.1. Let $a, d \in S$. An element a is left invertible along $d$ if there exists $b \in S$ such that bad $=d$ and $b \leq_{\mathcal{L}} d$.

Any $b$ satisfying the conditions in Definition 2.1 is called a left inverse of $a$ along $d$.

Definition 2.2. Let $a, d \in S$. An element a is right invertible along $d$ if there exists $b$ such that $d a b=d$ and $b \leq_{\mathcal{R}} d$.

In [6], Mary defined a new generalized inverse in a semigroup as follows: An element $b$ is an inverse of $a$ along $d$ if $b a d=d=d a b$ and $b \leq_{\mathcal{H}} d$. This type inverse is unique, if it exists and denoted by $a^{\| d}$. Mary showed in particular that $a^{\#}, a^{D}$ and $a^{\dagger}$ are the inverses of $a$ along $a, a^{n}$ and $a^{*}$ respectively ([6, Theorem 11]). In [3], Drazin introduced $(b, c)$-inverse in a semigroup. It follows that $(d, d)$-inverse of $a$ is an inverse of $a$ along $d$ (Mary's inverse). Hence, group inverse, Drazin inverse, Moore-Penrose inverse and Mary's inverse of $a$ are instances of left or right inverse of $a$ along $d$.

Next, we present an existence criterion of a left inverse along an element.

Theorem 2.3. Let $a, d \in S$. Then a is left invertible along $d$ if and only if $d \leq_{\mathcal{L}}$ dad.

Proof. " $\Rightarrow$ " Suppose that $a$ is left invertible along $d$. Then there exists $b$ such that $b a d=d$ and $b \leq_{\mathcal{L}} d$. From $b \leq_{\mathcal{L}} d$, it follows that $b=x d$ for some $x \in S^{1}$. Hence, $d=b a d=x d a d$, which implies $d \leq_{\mathcal{L}} d a d$.

" $\Leftarrow " d \leq_{\mathcal{L}}$ dad implies $d=y d a d$ for some $y \in S$. Take $b=y d$. Then $b \leq_{\mathcal{L}} d$ and $b a d=d$.

Dually, we can obtain an equivalence for the existence of a right inverse along an element.

Theorem 2.4. Let $a, d \in S$. Then a is right invertible along $d$ if and only if $d \leq_{\mathcal{R}}$ dad.

Applying Theorems 2.3 and 2.4 and [7, Theorem 2.2], we get the following corollaries. 
Corollary 2.5. Let $a, d \in S$. Then a is invertible along $d$ if and only if it is left and right invertible along $d$.

Corollary 2.6. Let $d_{l}, d_{r}$ and $d$ be such that $S^{1} d_{l}=S^{1} d$ and $d_{r} S^{1}=d S^{1}$. Then $a$ is invertible along $d$ if and only if it is left invertible along $d_{l}$ and right invertible along $d_{r}$.

We consider now the relations between left invertibility along $d$ and left invertibility, left regularity, left $\pi$-regularity and left $*$-regularity.

Theorem 2.7. Let $a \in S$.

(i) If $S$ is a monoid, then a is left invertible along 1 if and only it is left invertible.

(ii) a is left invertible along $a$ if and only if it is left regular.

(iii) There exists $n \in \mathbb{N}$ such that a is left invertible along $a^{n}$ if and only if it is left $\pi$-regular.

(iv) If $S$ is a*-semigroup, then $a$ is left invertible along $a^{*}$ if and only if it is left *-regular.

Proof. (i) Suppose that $a$ is left invertible. Then there exists $b \in S$ such that $1=b a$. Also, as $b=b * 1$, then $b \leq_{\mathcal{L}} 1$ and $a$ is left invertible along 1 .

Conversely, if $a$ is left invertible along 1 , then there exists $b \in S$ such that $b a=1$ and $a$ is left invertible.

(ii) Assume that $a$ is left regular. Then exists $b$ in $S, a=b a^{2}$ hence $a=b^{2} a^{3}$ and $a \leq_{\mathcal{L}} a^{3}$. By Theorem 2.3, $a$ is left invertible along $a$.

Conversely, if $a$ is left invertible along $a$, then there is $b$ in $S$ such that $b a a=a$ and $a$ is left regular.

(iii) Let $a$ be left $\pi$-regular. Then there exist $b$ in $S$ and an integer $n$ such that $a^{n}=b a^{n+1}$, and by induction $a^{n}=b^{2} a^{n+2}=\cdots=b^{n+1} a^{2 n+1}$. Hence $a \leq_{\mathcal{L}} a^{2 n+1}$ and $a$ is left invertible along $a^{n}$ by Theorem 2.3.

The converse part is straightforward.

(iv) Assume that $a$ is left $*$-regular. Then there exists $x \in S$ such that $a=a a^{*} a x$ and hence $a^{*}=x^{*} a^{*} a a^{*}$, which implies that $a$ is left invertible along $a^{*}$ by Theorem 2.3 .

Conversely, if $a$ is left invertible along $a^{*}$, it follows from Theorem 2.3 that $a^{*} \leq_{\mathcal{L}} a^{*} a a^{*}$. Hence, $a=a a^{*} a y$ for some $y \in S$ and $a$ is left $*$-regular. 
Applying Theorems 2.3 and 2.7, we give some characterizations of left invertibility and left generalized invertibilities in the following corollary.

Corollary 2.8. Let $a \in S$. Then

(i) If $S$ is a monoid, a is left invertible if and only if $1 \leq_{\mathcal{L}}$ a.

(ii) $a$ is left regular if and only if $a \leq_{\mathcal{L}} a^{3}$.

(iii) $a$ is left $\pi$-regular if and only if $a^{m} \leq_{\mathcal{L}} a^{2 m+1}$, for a positive integer $m$.

(iv) If $S$ is a*-semigroup, then a is left *-regular if and only if $a^{*} \leq_{\mathcal{L}}$ $a^{*} a a^{*}$.

Dually, we have the following result.

Theorem 2.9. Let $a \in S$. Then

(i) If $S$ is a monoid, a is right invertible along 1 if and only it is right invertible.

(ii) a is right invertible along $a$ if and only if it is right regular.

(iii) $a$ is right invertible along $a^{m}$ if and only if it is right $\pi$-regular.

(iv) If $S$ is a *-semigroup, then a is right invertible along $a^{*}$ if and only if it is right $*$-regular.

By Theorems 2.4 and 2.9, we have

Corollary 2.10. Let $a \in S$. Then

(i) If $S$ is a monoid, $a$ is right invertible if and only if $1 \leq_{\mathcal{R}} a$.

(ii) $a$ is right regular if and only if $a \leq_{\mathcal{R}} a^{3}$.

(iii) $a$ is right $\pi$-regular if and only if $a^{m} \leq_{\mathcal{R}} a^{2 m+1}$, for a positive integer $m$.

(iv) If $S$ is a *-semigroup, then a is right $*$-regular if and only if $a^{*} \leq_{\mathcal{R}}$ $a^{*} a a^{*}$.

Remark 2.11. Let $S$ be a non Dedekind finite ring with $a b=1 \neq b a$. Then $a$ is right invertible along $a\left(a^{n}\right)$ by Theorem 2.4 , but one can show that it is not left invertible along $a\left(a^{n}\right)$. However, in a $*$-semigroup, we prove that every right $*$-regular element is left $*$-regular (see Theorem 2.16 below).

We present characterizations of $\{1,3\}$-inverse, $\{1,4\}$-inverse, left $*$-regularity and right $*$-regularity of an element in a $*$-semigroup with an identity element.

The conditions (i) and (ii) in Proposition 2.12 below were essentially proved in [11, Lemma 2.2] in a ring with involution case. 
Proposition 2.12. Let $S$ be a *-semigroup and let $a \in S^{1}$. Then

(i) a has a $\{1,3\}$-inverse if and only if $S^{1} a=S^{1} a^{*} a$.

(ii) a has a $\{1,4\}$-inverse if and only if $a S^{1}=a a^{*} S^{1}$.

(iii) $a$ is left *-regular if and only if $a S^{1}=a a^{*} a S^{1}$.

(iv) $a$ is right $*$-regular if and only if $S^{1} a=S^{1} a a^{*} a$.

Remark 2.13. Proposition 2.12 does not hold in the case that there is no identity element. Indeed, let $S$ be a null semigroup $(x y=0, \forall x, y \in S)$ distinct from $\{0\}$. Then 0 is the only von Neumann regular element but $(\forall a \in S) S a=0=S a a^{*} a=S a^{*} a$ for instance.

Remark 2.14. If $a$ is left $*$-regular, then $a$ has a $\{1,4\}$-inverse by Proposition 2.12. But the converse does not necessarily hold. Let $S=M_{2}(\mathbb{C})$ and the involution is the transpose. Take $A=\left(\begin{array}{ll}1 & 0 \\ i & 0\end{array}\right)$ and $A^{*}=\left(\begin{array}{ll}1 & i \\ 0 & 0\end{array}\right)$. Then $A A^{*} S=A S$, which implies that $A$ is $\{1,4\}$-invertible. However $A A^{*} A S=0$. So, $A$ is not left $*$-regular.

Now, we construct a $*$-semigroup to illustrate various relations in Proposition 2.12 .

Example 2.15. Let $A=\{1,2,3\}$. Then every map from $A$ to $A$ can be written as $\left(\begin{array}{lll}1 & 2 & 3 \\ i & j & k\end{array}\right)$, where $i, j, k \in A$. If $S$ is a semigroup generated by $x=\left(\begin{array}{lll}1 & 2 & 3 \\ 2 & 3 & 3\end{array}\right)$ and $y=\left(\begin{array}{lll}1 & 2 & 3 \\ 1 & 1 & 3\end{array}\right)$, then $S=\left\{x, x^{2}, y, x y, y x\right\}$. Set $x^{*}=x,\left(x^{2}\right)^{*}=x^{2}, y^{*}=y,(x y)^{*}=y x$ and $(y x)^{*}=x y$, then $*$ is an involution on $S$. Moreover, we get

(i) $x$ is regular but neither $\{1,3\}$ nor $\{1,4\}$-invertible.

(ii) $y$ and $x^{2}$ are projectors and hence Moore-Penrose invertible.

(iii) $x y$ is $\{1,4\}$-invertible but neither $\{1,3\}$-invertible nor left $*$-regular.

(iv) $y x$ is $\{1,3\}$-invertible but neither $\{1,4\}$-invertible nor right $*$-regular.

Theorem 2.16. Let $S$ be $a *$-semigroup and let $a \in S$. Then the following conditions are equivalent:

(i) a is Moore-Penrose invertible.

(ii) a is left $*$-regular.

(iii) a is right $*$-regular. 
ProOF. (i) $\Rightarrow$ (ii) Let $a^{\dagger}$ be the Moore-Penrose inverse of $a$. Then $a=$ $a\left(a^{\dagger} a\right)^{*}=a a^{*}\left(a^{\dagger} a a^{\dagger}\right)^{*}=a a^{*} a a^{\dagger}\left(a^{\dagger}\right)^{*}$ and hence $a$ is left $*$-regular.

(ii) $\Leftrightarrow$ (iii) Assume that $a$ is left $*$-regular. There exists $x \in S$ such that $a=a a^{*} a x$ and hence $a^{*}=x^{*} a^{*} a a^{*}$. Since $(a x)^{*} a=(a x)^{*} a a^{*}(a x)$, it follows that $(a x)^{*} a=\left[(a x)^{*} a\right]^{*}=a^{*} a x$. Hence, we have $a=a a^{*} a x=a(a x)^{*} a=$ $a x^{*} a^{*} a=\left(a x^{*} x^{*} a^{*}\right) a a^{*} a$. So, $a$ is right $*$-regular.

The converse part follows by a similar way.

(iii) $\Rightarrow$ (i) Let $a$ be right $*$-regular and hence left $*$-regular. We have $a \in$ $a a^{*} S \cap S a^{*} a$. Thus, $a$ is Moore-Penrose invertible.

Recall that a semigroup $S$ is called *-regular if all elements in $S$ are Moore-Penrose invertible. Hence, we get

Corollary 2.17. Let $S$ be a *-semigroup. Then $S$ is *-regular if and only every element in $S$ is left (right) *-regular.

The following lemma was given by Penrose in complex matrices (see $[9$, p. 407]), it indeed holds in a *-semigroup.

Lemma 2.18. Let $S$ be $a *$-semigroup and let $a \in S$. If $a x a=a=a y a$, $(a x)^{*}=a x$ and $(y a)^{*}=y a$ for some $x, y \in S$. Then a is Moore-Penrose invertible and $a^{\dagger}=$ yax.

We now present the formula of the Moore-Penrose inverse of a left (right) *-regular element.

Theorem 2.19. Let $S$ be $a$ *-semigroup and let $a \in S$. If $a=a a^{*} a x$ for some $x \in S$, then a is Moore-Penrose invertible and $a^{\dagger}=a^{*} a x^{2} a^{*}$.

Proof. If $a=a a^{*} a x$, then $(a x)^{*}$ is a $\{1,4\}$-inverse of $a$ according to [11, Lemma 2.2]. By the proof (ii) $\Leftrightarrow$ (iii) in Theorem 2.16, it is known that $a=\left(a x^{*} x^{*} a^{*} a\right) a^{*} a$, and $\left(a x^{*} x^{*} a^{*} a\right)^{*}$ is a $\{1,3\}$-inverse of $a$. By virtue of Lemma 2.18, it follows that $a$ is Moore-Penrose invertible and $a^{\dagger}=$ $(a x)^{*} a\left(a x^{*} x^{*} a^{*} a\right)^{*}=a^{*} a x^{2} a^{*}$.

Dually, we have the following result.

Theorem 2.20. Let $S$ be $a *$-semigroup and let $a \in S$. If $a=y a a^{*} a$ for some $y \in S$, then $a$ is Moore-Penrose invertible and $a^{\dagger}=a^{*} y^{2} a a^{*}$. 
We then recover and improve some known characterizations of generalized invertibility in a semigroup.

Corollary 2.21. [6, Theorem 11] Let $a \in S$. Then

(i) a is invertible if and only if it is invertible along 1. In this case, $a^{-1}=a^{\| 1}$.

(ii) a is group invertible if and only if it is invertible along a. In this case, $a^{\#}=a^{\| a}$.

(iii) a is Drazin invertible if and only if there exists an integer $n$, $a$ is invertible along $a^{n}$. In this case, $a^{D}=a^{\| a^{n}}$.

(iv) a is Moore-Penrose invertible if and only if it is left (right) invertible along $a^{*}$. In this case, $a^{\dagger}=a^{\| a^{*}}$.

\section{One-sided inverse along a product in rings}

In this section, we present equivalent conditions for the existence of onesided inverse along a product in a ring $R$. In what follows, $R$ is always an associative ring with unity 1.

First, we begin with a well-known lemma.

Lemma 3.1. Let $a, b, c \in R$.

(i) If $(1+a b) c=1$, then $(1+b a)(1-b c a)=1$.

(ii) If $c(1+a b)=1$, then $(1-b c a)(1+b a)=1$.

It follows from Lemma 3.1 that $1+a b$ is (left, right) invertible if and only if $1+b a$ is (left, right) invertible and $(1+b a)^{-1}=1-b(1+a b)^{-1} a$. This result is known as Jacobson's Lemma.

Let $a \in R$. By $a_{l}^{-1}$ and $a_{r}^{-1}$ we denote a left inverse and a right inverse of $a$, respectively. Next, we present an existence criterion of a left inverse along a product by means of one-sided invertibility of certain elements.

Theorem 3.2. Let $p, a, q, m \in R$ with $m$ regular. If $m \leq_{\mathcal{L}}$ pm and $m \leq_{\mathcal{R}}$ $m q$, then the following conditions are equivalent:

(i) a is left invertible along pmq.

(ii) $u=m q a p+1-m m^{(1)}$ is left invertible.

(iii) $v=$ qapm $+1-m^{(1)} m$ is left invertible.

In this case, $p u_{l}^{-1} m q$ is a left inverse of a along pmq. 
Proof. It follows from Lemma 3.1 that (ii) $\Leftrightarrow$ (iii).

(i) $\Rightarrow$ (ii) Suppose that $a$ is left invertible along $p m q$. From Theorem 2.3, we get $p m q \leq_{\mathcal{L}}$ pmqapmq. Hence, there exists $x \in R$ such that

$$
p m q=x p m q a p m q .
$$

By $m \leq_{\mathcal{R}} m q$, there exists $q^{\prime} \in R$ such that $m=m q q^{\prime}$. Similarly, $m \leq_{\mathcal{L}} p m$ guarantees that $m=p^{\prime} p m$ for some $p^{\prime} \in R$. Multiplying the equation $(*)$ by $q^{\prime}$ on the right yields $p m=x p m q a p m$. Set $y=m m^{(1)} p^{\prime} x p m m^{(1)}+1-m m^{(1)}$, we obtain $y\left(m q a p m m^{(1)}+1-m m^{(1)}\right)=1$. Indeed, we have

$$
\begin{aligned}
& y\left(m q a p m m^{(1)}+1-m m^{(1)}\right) \\
= & \left(m m^{(1)} p^{\prime} x p m m^{(1)}+1-m m^{(1)}\right)\left(m q a p m m^{(1)}+1-m m^{(1)}\right) \\
= & m m^{(1)} p^{\prime} x p m q a p m m^{(1)}+1-m m^{(1)} \\
= & m m^{(1)} p^{\prime} p m m^{(1)}+1-m m^{(1)} \\
= & m m^{(1)}+1-m m^{(1)} \\
= & 1 .
\end{aligned}
$$

Consequently, mqapmm $^{(1)}+1-m m^{(1)}$ is left invertible. Again, Lemma 3.1 ensures that mqap $+1-m m^{(1)}$ is left invertible.

(ii) $\Rightarrow$ (i) Suppose now that $u$ is left invertible. Then there is $u^{\prime}$ such that $u^{\prime} u=1$. Since $u m=m q a p m$, it follows that $m=u^{\prime} m q a p m$. Also, by $m \leq_{\mathcal{L}} p m$, there exists $p^{\prime} \in R$ such that $p^{\prime} p m=m$ and hence $p m q=$ $p u^{\prime} m q a p m q=p u^{\prime} p^{\prime} p m q a p m q$. Take $b=p u^{\prime} p^{\prime} p m q$, then $b \leq_{\mathcal{L}} p m q$, that is, $a$ is left invertible along $p m q$.

Hence, $b=p u_{l}^{-1} m q$ is a left inverse of $a$ along $p m q$.

As a special corollary of Theorem 3.2, we get

Corollary 3.3. Let $a, m \in R$ with $m$ regular. Then the following conditions are equivalent:

(i) a is left invertible along $m$.

(ii) $u=m a+1-m m^{(1)}$ is left invertible.

(iii) $v=a m+1-m^{(1)} m$ is left invertible.

In this case, $u_{l}^{-1} m$ is a left inverse of a along $m$.

Dually, we have 
Theorem 3.4. Let $p, a, q, m \in R$ with $m$ regular. If $m \leq_{\mathcal{L}} p m$ and $m \leq_{\mathcal{R}}$ $m q$, then the following conditions are equivalent:

(i) $a$ is right invertible along pmq.

(ii) $u=m q a p+1-m m^{(1)}$ is right invertible.

(iii) $v=q a p m+1-m^{(1)} m$ is right invertible.

In this case, $p m v_{r}^{-1} q$ is a right inverse of a along $p m q$.

Corollary 3.5. Let $a, m \in R$ with $m$ regular. Then the following conditions are equivalent:

(i) $a$ is right invertible along $m$.

(ii) $u=m a+1-m m^{(1)}$ is right invertible.

(iii) $v=a m+1-m^{(1)} m$ is right invertible.

In this case, $m v_{r}^{-1}$ is a right inverse of a along $m$.

An involution $*$ in a ring $R$ is an anti-isomorphism of degree 2 which satisfies $\left(a^{*}\right)^{*}=a,(a b)^{*}=b^{*} a^{*}$ and $(a+b)^{*}=a^{*}+b^{*}$, for all $a, b \in R$.

Let $S$ be a ring with involution in Theorem 2.16. We have

Corollary 3.6. Let $R$ be a ring with involution and let $a \in R$. Then

(i) $a$ is left *-regular if and only if it is right $*$-regular.

(ii) $R$ is $*$-regular if and only if every element in $R$ is left (right) $*$-regular.

Recall that a ring $R$ is called strongly $\pi$-regular if each element $a \in R$ is left (right) $\pi$-regular (see e.g. [1]). In particular, $R$ is called strongly regular if each element $a \in R$ is left (right) regular. We next give new characterizations of strongly $(\pi-)$ regular rings, $*$-regular rings, by one-sided invertibility along an element.

Corollary 3.7. Let $a \in R$. Then

(i) $R$ is a strongly regular ring if and only if every element a is left (right) invertible along a.

(ii) $R$ is a strongly $\pi$-regular ring if and only if every element a is left (right) invertible along $a^{n}$ for some positive $n$.

(iii) $R$ is a *-regular ring if and only if every element a is left (right) invertible along $a^{*}$.

We have seen that $a$ is both left and right invertible along $p m q$ if and only if it is invertible along $p m q$. Moreover, the inverse of $a$ along $p m q$ is unique (Corollary 2.5). Hence we have 
Corollary 3.8. ([10, Theorem 2.2] Let $p, a, q, m \in R$ with $m$ regular. If $m \leq_{\mathcal{L}} p m$ and $m \leq_{\mathcal{R}} m q$, then the following conditions are equivalent:

(i) $a^{\| p m q}$ exists.

(ii) $u=m q a p+1-m m^{(1)}$ is invertible.

(iii) $v=q a p m+1-m^{(1)} m$ is invertible.

In this case,

$$
a^{\| p m q}=p u^{-1} m q=p m v^{-1} q .
$$

By taking $p=q=1$ we get

Corollary 3.9. ([7, Theorem 3.2] and [8, Theorem 1.3]) Let $m \in R$ be regular. Then the following are equivalent:

(i) $a$ is invertible along $m$.

(ii) $u=m a+1-m m^{(1)}$ is invertible.

(iii) $v=a m+1-m^{(1)} m$ is invertible.

In this case,

$$
a^{\| m}=u^{-1} m=m v^{-1} .
$$

We finally give some applications of the inverse along a product by its existence criterion. More results on the inverse along a matrix can be referred to references $[8,10]$. By the symbol $R_{2 \times 2}$ we denote the ring of $2 \times 2$ matrices over a ring $R$.

Let $A=\left[\begin{array}{ll}a & c \\ b & d\end{array}\right], D=\left[\begin{array}{ll}d_{1} & d_{3} \\ d_{2} & d_{4}\end{array}\right] \in R_{2 \times 2}$ with $D$ regular and assume that $d_{4}$ in matrix $D$ is invertible. Then we have the following decomposition

$$
D=\left[\begin{array}{ll}
d_{1} & d_{3} \\
d_{2} & d_{4}
\end{array}\right]=\left[\begin{array}{cc}
1 & d_{3} d_{4}^{-1} \\
0 & 1
\end{array}\right]\left[\begin{array}{cc}
s & 0 \\
0 & d_{4}
\end{array}\right]\left[\begin{array}{cc}
1 & 0 \\
d_{4}^{-1} d_{2} & 1
\end{array}\right]=: P M Q,
$$

where $s=d_{1}-d_{3} d_{4}^{-1} d_{2}$ is the Schur complement of $d_{4}$ in the matrix $D$. It is well known that $D$ is regular if and only if $M$ is regular. Similarly, if $d_{1}$ is invertible, $d_{4}-d_{2} d_{1}^{-1} d_{3}$ is called the Schur complement of $d_{1}$ in the matrix $D$.

According to Corollary 3.8, it is known that $A^{\| D}$ exists if and only if $U=$ $M Q A P+I-M M^{(1)}$ is invertible. One can get $I-M M^{(1)}=\left[\begin{array}{cc}1-s s^{(1)} & 0 \\ 0 & 0\end{array}\right]$ by a direct calculation. 
We also get that $M Q A P=\left[\begin{array}{cc}s a & \alpha \\ d_{2} a+d_{4} b & \beta\end{array}\right]$, where

$$
\begin{aligned}
\alpha & =s\left(a d_{3} d_{4}^{-1}+c\right), \\
\beta & =\left(d_{2} a+d_{4} b\right) d_{3} d_{4}^{-1}+d_{2} c+d_{4} d
\end{aligned}
$$

Hence, it follows that $U=\left[\begin{array}{cc}u & \alpha \\ d_{2} a+d_{4} b & \beta\end{array}\right]$, where $u=s a+1-s s^{(1)}$. If $a^{\| s}$ exists, applying Corollary 3.9, it follows that $u$ is invertible.

Using the Schur complement, we have

$$
U=\left[\begin{array}{cc}
u & \alpha \\
d_{2} a+d_{4} b & \beta
\end{array}\right]=\left[\begin{array}{cc}
1 & 0 \\
\left(d_{2} a+d_{4} b\right) u^{-1} & 1
\end{array}\right]\left[\begin{array}{ll}
u & 0 \\
0 & \xi
\end{array}\right]\left[\begin{array}{cc}
1 & u^{-1} \alpha \\
0 & 1
\end{array}\right],
$$

where $\xi=\beta-\left(d_{2} a+d_{4} b\right) a^{\| s}\left(a d_{3} d_{4}^{-1}+c\right)$. Moreover, $U$ is invertible if and only if $\xi$ is invertible.

In this case,

$$
U^{-1}=\left[\begin{array}{cc}
1 & -u^{-1} \alpha \\
0 & 1
\end{array}\right]\left[\begin{array}{cc}
u^{-1} & 0 \\
0 & \xi^{-1}
\end{array}\right]\left[\begin{array}{cc}
1 & 0 \\
-\left(d_{2} a+d_{4} b\right) u^{-1} & 1
\end{array}\right]
$$

Thus, $A^{\| D}$ exists if and only if $\xi=\beta-\left(d_{2} a+d_{4} b\right) a^{\| s}\left(a d_{3} d_{4}^{-1}+c\right)$ is invertible. Moreover, we get

$$
\begin{aligned}
A^{\| D} & =P U^{-1} M Q=\left[\begin{array}{cc}
x_{1} s+x_{3} d_{2} & x_{3} d_{4} \\
x_{2} s+\xi^{-1} d_{2} & \xi^{-1} d_{4}
\end{array}\right], \text { where } \\
x_{1} & =u^{-1}+\left(u^{-1} \alpha-d_{3} d_{4}^{-1}\right) \xi^{-1}\left(d_{2} a+d_{4} b\right) u^{-1} \\
x_{2} & =-\xi^{-1}\left(d_{2} a+d_{4} b\right) u^{-1} \\
x_{3} & =d_{3} d_{4} \xi^{-1}-u^{-1} \alpha \xi^{-1} .
\end{aligned}
$$

Remark 3.10. Even if $a^{\| s}$ does not exist, $A^{\| D}$ may exist. For instance, take $A=\left[\begin{array}{ll}a & c \\ b & d\end{array}\right]=\left[\begin{array}{ll}0 & 1 \\ 1 & 0\end{array}\right], D=\left[\begin{array}{ll}d_{1} & d_{3} \\ d_{2} & d_{4}\end{array}\right]=\left[\begin{array}{ll}1 & 0 \\ 0 & 1\end{array}\right] \in R_{2 \times 2}$. Since $s=$ $d_{1}-d_{3} d_{4}^{-1} d_{2}=1$, it follows that $s a+1-s s^{(1)}=0$. Hence, $a^{\| s}$ does not exist by Corollary 3.9. However, $A$ is invertible along $D$ since they are both invertible. 
We close this section with some further remarks:

(i) In Theorem 3.2, since $v_{l}^{-1}\left(1+\left(q a p-m^{(1)}\right) m\right)=1$, it follows that $1-m v_{l}^{-1}\left(q a p-m^{(1)}\right)$ is a left inverse of $u$ by Lemma 3.1. Hence, we can give the representation of a left inverse of $a$ along $p m q$ by $v_{l}^{-1}$.

(ii) We give another proof for Corollary 3.6(i). Assume that $a$ is left $*-$ regular (we have $a=a a^{*} a x$ for some $x \in R$ ). Then it is left invertible along $a^{*}$ according to Theorem 2.7. Moreover, $a$ is regular, and $(a x)^{*}$ is an inner inverse (indeed a $\{1,4\}$-inverse) of $a$. Indeed, it follows that $\left[(a x)^{*} a\right]^{*}=a^{*} a x=$ $(a x)^{*} a$ and $a(a x)^{*} a=a a^{*} a x=a$ since $a^{*} a x=\left(a a^{*} a x\right)^{*} a x=(a x)^{*} a a^{*} a x=$ $(a x)^{*} a$. By Corollary 3.3, $u=a^{*} a+1-a^{*}\left(a^{*}\right)^{(1)}=a^{*} a+1-\left(a^{(1)} a\right)^{*}$ is left invertible. Hence, we can pick an inner inverse $(a x)^{*}$ of $a$ such that $a^{(1)} a$ is symmetric. Then $u=u^{*}$ is right invertible, and by Corollary 3.5, it follows that $a$ is right invertible along $a^{*}$.

\section{ACKNOWLEDGMENTS}

The authors are highly grateful to the referee for valuable comments which led to improvements of this paper. In particular, Corollaries 2.5, 2.6 and 3.6, Remarks 2.13 and 3.10 and the final remark (ii) were suggested to the authors by the referee. The first author is grateful to China Scholarship Council for giving him a purse for his further study in University of Minho, Portugal. Jianlong Chen and Huihui Zhu are financed by the National Natural Science Foundation of China (No. 11201063 and No. 11371089), the Specialized Research Fund for the Doctoral Program of Higher Education (No. 20120092110020), the Natural Science Foundation of Jiangsu Province (No. BK20141327), the Foundation of Graduate Innovation Program of Jiangsu Province(No. CXLX13-072), the Scientific Research Foundation of Graduate School of Southeast University and the Fundamental Research Funds for the Central Universities (No. 22420135011). Pedro Patrício is financed by the Research Centre of Mathematics of the University of Minho with the Portuguese Funds from the "Fundação para a Ciência e a Tecnologia", through the Project PEst-OE/MAT/UI0013/2014.

\section{References}

[1] P. Ara, Strongly $\pi$-regular rings have stable range one, Proc. Amer. Math. Soc. 1996;124:3293-3298. 
[2] G. Azumaya, Strongly $\pi$-regular rings, J. Fac. Sci. Hokkaido Univ. 1954;13:34-39.

[3] M.P. Drazin, A class outer generalized inverses, Linear Algebra Appl. 2012;436:1909-1923.

[4] M.P. Drazin, Pseudo-inverses in associative rings and semigroups, Amer. Math. Monthly 1958;65:506-514.

[5] J.A. Green, On the structure of semigroups, Ann. Math. 1951;54:163172.

[6] X. Mary, On generalized inverses and Green's relations, Linear Algebra Appl. 2011;434:1836-1844.

[7] X. Mary, P. Patrício, Generalized inverses modulo $\mathcal{H}$ in semigroups and rings, Linear Multilinear Algebra 2013;61:886-891.

[8] X. Mary, P. Patrício, The inverse along a lower triangular matrix, Appl. Math. Comput. 2012;219:1130-1135.

[9] R. Penrose, A generalized inverse for matrices, Proc. Camb. phil. Soc. 1955;51:406-413.

[10] H.H. Zhu, P. Patrício, J.L. Chen, The inverse along a product and its applications, arXiv:1411.7847v1 [math.RA].

[11] H.H. Zhu, X.X. Zhang, J.L. Chen, Generalized inverses of a factorization in a ring with involution, Linear Algebra Appl. 2015;472:142-150. 